\title{
Validation of a Spanish Chronic Obstructive Sialadenitis Quality of Life Questionnaire
}

\author{
Jessica Mireya Santillán Coelloํ․ Alvaro Sanchez Barrueco ${ }^{1}$, Fernando González Galán², \\ Gonzalo Diaz Tapia ${ }^{2}$, Ignacio Mahillo Fernández ${ }^{3}$, Carlos Cenjor Español ${ }^{4}$, and José \\ Miguel Villacampa Aubáa \\ ${ }^{1}$ Universidad Alfonso X el Sabio \\ ${ }^{2}$ Hospital General de Villalba \\ ${ }^{3}$ Instituto de Investigacion Sanitaria de la Fundacion Jimenez Diaz \\ ${ }^{4}$ Hospital Universitario Fundacion Jimenez Diaz
}

January 24, 2022

\begin{abstract}
Objectives: To design a Spanish questionnaire to assess quality of life in chronic obstructive sialadenitis (COS), named CSOC and assess its reliability, validity and feasibility. Design: A prospective, multicentre, observational study Settings: Salivary Gland Disorder and Sialendoscopy Unit, University Tertiary Hospital. Participants: Patients with diagnosis of COS and indication for sialendoscopy were included in the study. Main outcome measures: The item generation process included a review of published data as well as interviews with patients. An expert panel then tested the content validity of the instrument and the construct validity was tested in 120 patients. Patients completed a self-administered CSOC questionnaire, a Short Form-36 and a Visual Analogue Scale. Feasibility, reliability, internal consistency, construct validity and responsiveness were assessed. Results: All the patients found the instrument understandable. Cronbach $\alpha$ coefficient was high (0.85). The time required to fill out was 5.7 and 4.5 minutes for pre and postsialendoscopy CSOC respectively. Cronbach $\alpha$ coefficient was very high for both pre and postsialendoscopy CSOC (0.90 and 0.94 respectively). The correlation with the SF-36 dimensions was negative and positive with the VAS. The mean score of CSOC was 28.6 and 7.98 for pre and postsialendoscopy Conclusions: The CSOC questionnaire is understandable, feasible, reliable and representative of quality of life in COS. Keywords: sialadenitis, QOL, questionnaire, validation, sialendoscopy
\end{abstract}

Abstract

Objectives: To design a Spanish questionnaire to assess quality of life in chronicobstructive sialadenitis (COS), named CSOC and assess its reliability, validity andfeasibility.

Design : A prospective, multicentre, observational study

Settings: Salivary Gland Disorder and Sialendoscopy Unit, University Tertiary Hospital.

Participants: Patients with diagnosis of COS and indication for sialendoscopy were included in the study.

Main outcome measures: The item generation process included a review of published data as well as interviews with patients. An expert panel then tested the content validity of theinstrument and the construct validity was tested in 120 patients. Patients completed a self-administered CSOC questionnaire, a Short Form-36 and a Visual Analogue Scale. Feasibility, reliability, internal consistency, construct validity and responsiveness were assessed. 
Results: All the patients found the instrument understandable. Cronbach $\alpha$ coefficient was high (0.85). The time required to fill out was 5.7 and 4.5 minutes for pre and postsialendoscopy CSOC respectively. Cronbach $\alpha$ coefficient was very high for both pre and postsialendoscopy CSOC (0.90 and 0.94 respectively). The correlation with the SF-36 dimensions was negative and positive with the VAS. The mean score of CSOC was 28.6 and 7.98 for pre and postsialendoscopy

Conclusions: The CSOC questionnaire is understandable, feasible, reliable and representative of quality of life in COS.

\section{Key points:}

- Chronic obstructive sialadenitis causes a marked loss in the quality of life.

- Chronic obstructive sialadenitis and the results of sialendoscopy should be able to be evaluated by an objective tool.

- Sialendoscopy, when possible, is currently the ideal treatment in chronic obstructive sialadenitis and the first therapeutic recommendation.

- At this time, no statistically validated quality of life questionnaire applied to chronic obstructive sialadenitis has been described.

- The first statistically validated quality of life questionnaire applied to the chronic obstructive sialadenitis and results of sialendoscopy is presented.

\section{Introduction}

Chronic obstructive sialadenitis (COS) is a benign disorder characterized by repeated episodes of inflammation of the major salivary glands[1-3]. Clinical presentation includes painful swelling of the affected gland, usually trigged by meals or other means of salivary stimulation. After years in which the disorder received scant attention in research, the last decades have seen a growth in interest in the results of minimally invasive treatment with sialendoscopy.

Patient-reported outcomes (PROs) can simplify patient involvement in decision-making and may help healthcare professionals identify patientconcerns[4]. PROs are also essential indicators in clinical research and are frequently used as study endpoints[5]. Therefore, there is an unmet need to develop a specific PRO to assess $\operatorname{COS}[6]$.

To our knowledge, there are no existing Spanish questionnaires with which to assess quality of life (QOL) in COS. We identified 3 English-language questionnaires specifically designed for COS and sialendoscopy: Chronic Obstructive Sialadenitis Symptoms (COSS) [2, 7-10], Modified Oral Health Impact Profile-14 (OHIP14)[11], and patient satisfaction questionnaire[12].

We are aware that for a QOL questionnaire to be used in clinical practice or research, the instrument must be rigorously validated in order to avoid incorrect interpretations.

Our purpose is to develop and validate a questionnaire to assess QOL in COS - called CSOC (Spanish acronym for Cuestionario sobre Calidad de vida en Sialoadenitis Obstructiva Crónica ). The overall goal of CSOC is to assess the impact of COS on QOL and the extent to which QOL improves with sialendoscopy.

\section{Materials and Methods}

The study design and manuscript followed the STROBE reporting guideline.

\section{CSOC Questionnaire Development}

The developmental stage of a questionnaire consists of domain identification, item generation, and instrument construction[13]. Domain identification was accomplished by reviewing the published literature. Four papers made reference to specific questionnaires (COSS questionnaire[2, 7], modified OHIP-14[11], and patient satisfaction questionnaire[12]) and 3 analysed findings generated by applying generic QOL questionnaires (Glasgow Benefit Inventory[6, 14] and 5D-HRQoL[15]). 
We chose health-related QOL as our domain of interest. The conceptual definition of QOL selected was the level of well-being resulting from a self-assessment of several dimensions of life and considering the impact of these dimensions on health status[16]. The selected dimensions were physical function and symptoms, performance of social roles, occupational activities, emotional status, and how the patient feels about their health.

The next step was to generate a list of potential items for each dimension. After interviewing patients with COS, the research team selected 20 items. Items were organized from specific conditions related with sialadenitis to complaints related to overall health status. Details of the CSOC questionnaire are shown in table 1.

Each item uses a Likert-type response scale (score 0-4). Lower scores indicate better QOL in COS, whereas higher scores are suggestive of greater degrees of impairment (Supplementary Table 1).

At the start of the questionnaire, patients were asked to score each item based on their experience over the 3 preceding months. The research group used this timeframe because of the intermittent and fluctuating nature of COS symptoms according to their own experience.

\section{Study Population}

The "Blinded for review" approved all study proceedings (code Blinded for review ).

In the pilot study,the target for total enrolment was 30 participants in order to achieve a reasonably high power $(80 \%)$, to detect a problem that occurs in $5 \%$ of the population, and pinpoint a repeat occurrence of a problem that affects $10 \%$ of the respondents[17]. Convenience sampling was used to recruit subjects older than 18 years of age among those patients with diagnosis of COS by an otolaryngologist and indication for sialendoscopy evaluated at the department of otorhinolaryngology at "Blinded for review " between June 2017 and March 2018. COS was defined as at least 2 episodes of salivary gland swelling and discomfort in the previous 3 months. We excluded patients with a previous diagnosis of salivary glands tumour and those with insufficient proficiency in the Spanish language.

Eight otolaryngologists experienced in sialadenitis management and sialendoscopy from different hospitals and cities in "Blinded for review "agreed to form part of the expert panel; members of the research group were excluded. The size of the committee of experts was determined based on recommendations contained in the literature[18].

One hundred twenty patients were recruited for the validation study. The sample size was calculated using the literature recommendationsof including at least 5 subjects per item and not fewer than 100 subjects[19]. The inclusion and exclusion criteria were the same used to assemble the sample for the pilot study. Patient were recruited between July 2018 and July 2020.

\section{Study Procedures}

Pilot study: After providing written informed consent, participants completed a self-administered CSOC questionnaire. After filling out the CSOC, each patient was asked how well they had understood each item.

Content validity: We sent an e-mail to the experts alongside an explanatory cover letter, reviewer instructions, definitions of terms, and a content-review questionnaire. The members of the panel responded by e-mail. In the content-review questionnaire, experts were asked to judge how representative the questionnaire items are of QOL in COS using a 4-point scale: $1=$ not representative, $2=$ somewhat representative, $3=$ quite representative and $4=$ highly representative. Experts were prompted to evaluate the clarity of item construction and wording. Finally, suggestions for addition or deletion of items were requested.

Validation process: After providing written informed consent, participants completed a self-administered CSOC questionnaire at the following time points:within45 days before sialendoscopy, in the preoperative area before sialendoscopy and 90-120 days after sialendoscopy. SF-36 and visual analogue scale (VAS)fordiscomfort 
were completed at the first and third time points. The CSOC filled out during the validation process comprised 18 items according to the results of the pilot study and the content validityassessment (Supplementary Table 3).

\section{End Points}

The primary end points were to establish the comprehensibility, feasibility, reliability, content,construct validity, and responsiveness of the CSOC.

\section{Statistical Analysis}

Pilot study: Comprehensibility was calculated as the percentage of patients who stated that the instrument was understandable. Feasibility was assessed by examining the number of missing responses on the returned questionnaires. Internal consistency analysis was performed with the Cronbach $\alpha$ coefficient.

A content validity index (CVI) based on expert ratings of relevance was used to quantify content validity. We calculated the CVI for each item (I-CVI) and for the overall scale (OS-CVI). The I-CVI was calculated as the number of experts giving a rating of either 3 or 4 , divided by the number of experts. To determine OS-CVI, we calculated the I-CVI of each item on the questionnaire and then computed the average I-CVI across items[18] (Supplementary Table 2).

Validation process: Feasibility was assessed by examining the number of missing responses on the returned questionnaires and the time required to complete the questionnaire in minutes.A reliability analysis was conducted, following the test-retest procedure. Internal consistency analysis was performed with the Cronbach $\alpha$ coefficient. Construct validity was assessed using known groups validity and convergent/divergent validity. The Mann-Whitney U test was used to test for statistically significant differences between two subgroups of frequency (fewer than 2 episodes and more than 4 episodes per week). Convergent/divergent validity was assessed by correlating CSOC scores with SF-36 and VAS using the Pearson correlation coefficient. Responsiveness was evaluated comparing CSOC results before and after sialendoscopy by paired sample t-test.

\section{Results}

Thirty participants were included in the pilot study. Sixty-three percent were female, and their ages ranged from 18 to 62 years (median, 48 years). Half of the patients (50\%) had completed university studies, and high school and primary school were the highest levels of education completed by $40 \%$ and $10 \%$, respectively. The number of missing items from the CSOC was 0.

All the patients found the instrument understandable (100\% comprehensibility). However, 5 patients expressed concern about items 14 and 15, as they believed they did not account for their individual circumstances. These items inquired about work and academic activities, and the patients who raised issues with these items were either unemployed or retired. As a result, the research team included recreational activities and basic activities of daily living in the language of these items. The internal reliability for the 20 items was high (Cronbach $\alpha$ coefficient $=0.85$ ). The Cronbach $\alpha$ coefficient showed no improvement when individual items were deleted, meaning that no items negatively affected the internal consistency of the CSOC.

Table 2 contains the results of the expert ratings of relevance for each item. Items 5 and 6 were given an I-CVI of 0.63 , meaning that they should be considered for elimination. Items 4,9 and 13 received an I-CVI of 0.75 , which indicates that further consideration should be given to these. After it was confirmed that eliminating these items did not affect internal consistency, the research group decided to exclude items 5 and 6. The members of the research group reviewed items 4, 9, and 13 (I-CV=0.75) and finally decided to maintain these in the second phase of the validation process. The OS-CVI was 0.85 and if we eliminate items 5 and 6 , the OS-CVI increases to 0.88 . One expert suggested eliminating item 5 , which also had a low I-CVI. The expert panel suggested adding 3 items: (1) Presence of purulent or thick saliva; (2) Difference between parotid and submandibular gland; and (3) Use of drugs to relieve symptoms. The research group decided not to incorporate these additional items. The presence of purulent or thick saliva is similar to item 
5, which was not considered to be representative. Differences noted in the affected gland was not considered appropriate within a QOL questionnaire. Finally, use of drugs to relieve the symptoms was included as part of item 18.

Once the CSOC was modified in light of analytical findings and expert feedback, the final version comprised 18 question (Supplementary Table 3) (total score 0-72).

One hundred and twenty subjects completed the presialendoscopy CSOC. Seventy-three percent were female, and their median age was 48.3 years. One hundred fifteen subjects (96\%) completed the postsialendoscopy CSOC during the follow-up examination. The number of missing items from the pre and postsialendoscopy CSOC was 0 . The time required tofill out the CSOC was 5.7 minutes (2-15 minutes) and 4.5 minutes (1-15 minutes) for pre and postsialendoscopy CSOC respectively.

The reliability determined by test-retest washigh (interclass correlation coefficient $=0.86,95 \%$ CI, 0.77-0.91). The internal consistency for the 18 items was very high for both pre and postsialendoscopy CSOC (Cronbach $\alpha$ coefficient $=0.90$ and 0.94 , respectively). Cronbach $\alpha$ coefficient showed no improvement when individual items were deleted.

Significant differences were observed when the total CSOC score was compared between 2 different groups of patients according with the frequency of sialadenitis episodes during a week (fewer than 2 episodes or more than 4 episodes).A greater difference was observed in postsialendoscopy CSOC (table 3).

Figure1 shows the correlation coefficient between total CSOC score and the different dimensions of SF-36 and VAS. The correlation with the SF-36 dimensions was negative meaning that lower values of CSOC are equal to higher values of SF-36. The correlation with VAS was positive.

The mean score for presialendoscopy CSOC was 28.6 (range, 3-61) and for postsialendoscopy CSOC it was 7.98 (range, $0-41$ ).

\section{Discussion}

In a recent literature research, we identified 2 specific English-language questionnaires used to assess QOL in patients with COS: modified OHIP-14[11] and (COSS)[2, 7-10], one questionnaire to assess patient satisfaction post sialendoscopy[12] and another questionnaire (Multidisciplinary Salivary Gland Society (MSGS) questionnaire) to quantify the burden of symptoms caused by xerostomia and sialadenitis[20].

The primary purpose of the present article was to design a specific questionnaire in Spanish to assess QOL in patients with COS (Supplementary Table 3) across six selected dimensions (physical function and symptoms, social roles, occupational activities, emotional status, and health perception). There are some similarities between items of the CSOC and the Modified OHIP-14, COSS and MSGS. The patient satisfaction questionnaire, on the other hand, is quite different[12];it contains 14 questions that cover pre-sialendoscopy symptoms, progress post-sialendoscopy, change in QOL, and overall patient satisfaction and does not evaluate social roles, sleep disorders, or emotional status[12]. The modified OHIP-14 was designed to measure self-reported functional limitation, discomfort, and disability in patients with salivary gland pathology[11]; the COSS examines sialadenitis symptoms, daily functions, emotional symptoms, sleeping and daily activities[7]; and the MSGSevaluatesxerostomia and sialadenitis symptoms as well as their frequency, intensity, and associated discomfort[20].

As we can see, all 5 specific questionnaires measure the presence of symptoms and functional limitations. The CSOC, modified OHIP-14[11], and COSS[7] include items that target social roles. The CSOC and modified OHIP- 14 assess emotional status. The CSOC includes recreational activities to cover the whole occupational dimension. The modified OHIP-14 has an item on work[11] that does not take unemployed or retired people into account, and the COSS prompts participants to provide information on daily activities in general[7]. The COSS and CSOC have an item evaluating sleep.

A complete validation process was conducted with a pilot study as well asassessment of content and construct validity. None of the existing studies describes a process of content validity such as ours, which includes an 
expert panel.

After the pilot study and assessment of content validity,the CSOC was modified to include 18 items. The analysis of the new 18-items psychometric properties suggested that CSOC has high feasibility (absence of missing responses and around 5 minutes required to fill out the questionnaire), high stability (test retest of 0.86), high internal consistency (Cronbach $\alpha$ coefficient of 0.90), and high responsiveness to change. The absence of missing responses and the short time required indicate that the responders found the CSOC to be easy to use. The significant difference between the scores of pre- and postsialendoscopy CSOC means that CSOC is a good tool to assess the improvement of COS after sialendoscopy. Additionally, CSOC proved its capacity to distinguish between patients with frequent and occasional episodes of sialadenitis.

On the other hand, CSOC had a low to moderate negative correlation with the different dimensions and component summaries of SF-36. The correlation has the expected direction, and the low correlation could be explained by the fact that SF-36 is not sensitive enough to capture the difference in COS symptoms $[2,7]$.

This study has three main limitations. First, we were unable to assess criterion validity due to the lack of a validated gold standard test. Second, we did not use a healthy control group. Third, the subjects used for the validation process only represent those patients who have an indication for sialendoscopy; to validate CSOC for all COS patients, a study which includes patients with and without indication for sialendoscopy should be conducted.

\section{Conclusion}

To date, there is no validated questionnaire that addresses QOL in patients with COS. Also, there is no specific Spanish questionnaire to assess QOL in COS. The CSOC questionnaire is an understandable, feasible, validated, and reliable instrument measuring QOL in COS.

\section{References}

1. Wang S, Marchal F, Zou Z, et al (2009) Classification and management of chronic sialadenitis of the parotid gland. J Oral Rehabil 36:2-8. https://doi.org/10.1111/j.1365-2842.2008.01896.x

2. Aubin-Pouliot A, Delagnes EA, Chang JL, Ryan WR (2016) Sialendoscopy-assisted surgery and the chronic obstructive sialadenitis symptoms questionnaire: A prospective study. The Laryngoscope 126:13431348. https://doi.org/10.1002/lary.25759

3. Saunders JR, Hirata RM, Jaques DA (1986) Salivary glands. Surg Clin North Am 66:59-81. https://doi.org/10.1016/s0039-6109(16)43829-6

4. Frost MH, Reeve BB, Liepa AM, et al (2007) What is sufficient evidence for the reliability and validity of patient-reported outcome measures? Value Health J Int Soc Pharmacoeconomics Outcomes Res 10 Suppl 2:S94-S105. https://doi.org/10.1111/j.1524-4733.2007.00272.x

5. Anthoine E, Moret L, Regnault A, et al (2014) Sample size used to validate a scale: a review of publications on newly-developed patient reported outcomes measures. Health Qual Life Outcomes 12:. https://doi.org/10.1186/s12955-014-0176-2

6. Ianovski I, Morton RP, Ahmad Z (2014) Patient-perceived outcome after sialendoscopy using the glasgow benefit inventory. The Laryngoscope 124:869-874. https://doi.org/10.1002/lary.24343

7. Aubin-Pouliot A, Delagnes EA, Eisele DW, et al (2016) The Chronic Obstructive Sialadenitis Symptoms Questionnaire to assess sialendoscopy-assisted surgery. The Laryngoscope 126:93-99. https://doi.org/10.1002/lary.25521

8. Ryan WR, Plonowska KA, Gurman ZR, et al (2019) One-Year symptom outcomes after sialolithiasis treatment with sialendoscopy-assisted salivary duct surgery. The Laryngoscope 129:396-402. https://doi.org/10.1002/lary.27398 
9. Delagnes EA, Aubin-Pouliot A, Zheng M, et al (2017) Sialadenitis without sialolithiasis: Prospective outcomes after sialendoscopy-assisted salivary duct surgery. The Laryngoscope 127:1073-1079. https://doi.org/10.1002/lary.26308

10. Delagnes EA, Zheng M, Aubin-Pouliot A, et al (2017) Salivary duct stenosis: Short-term symptom outcomes after sialendoscopy-assisted salivary duct surgery. The Laryngoscope 127:2770-2776. https://doi.org/10.1002/lary.26665

11. Gillespie MB, O'Connell BP, Rawl JW, et al (2015) Clinical and quality-of-life outcomes following gland-preserving surgery for chronic sialadenitis. The Laryngoscope 125:1340-1344. https://doi.org/10.1002/lary.25062

12. Kroll T, Finkensieper M, Sharma SJ, et al (2013) Short-term outcome and patient satisfaction after sialendoscopy. Eur Arch Oto-Rhino-Laryngol Off J Eur Fed Oto-Rhino-Laryngol Soc EUFOS Affil Ger Soc Oto-Rhino-Laryngol - Head Neck Surg 270:2939-2945. https://doi.org/10.1007/s00405-013-2418-z

13. Grant JS, Davis LL (1997) Selection and use of content experts for instrument development. Res Nurs Health 20:269-274. https://doi.org/10.1002/(sici)1098-240x(199706)20:3<269::aid-nur9>3.0.co;2-g

14. Meier BA, Holst R, Schousboe LP (2015) Patient-perceived benefit of sialendoscopy as measured by the Glasgow Benefit Inventory. The Laryngoscope 125:1874-1878. https://doi.org/10.1002/lary.25369

15. Jokela J, Saarinen R, Mäkitie A, et al (2019) Costs of sialendoscopy and impact on health-related quality of life. Eur Arch Otorhinolaryngol 276:233-241. https://doi.org/10.1007/s00405-018-5196-9

16. Urzúa M A, Caqueo-Urízar A (2012) Calidad de vida: Una revisión teórica del concepto. Ter Psicológica 30:61-71. https://doi.org/10.4067/S0718-48082012000100006

17. Perneger TV, Courvoisier DS, Hudelson PM, Gayet-Ageron A (2015) Sample size for pre-tests of questionnaires. Qual Life Res Int J Qual Life Asp Treat Care Rehabil 24:147-151. https://doi.org/10.1007/s11136014-0752-2

18. Polit DF, Beck CT, Owen SV (2007) Is the CVI an acceptable indicator of content validity? Appraisal and recommendations. Res Nurs Health 30:459-467. https://doi.org/10.1002/nur.20199

19. Morales Vallejo P Tamaño necesario de la muestra: ¿Cuántos sujetos necesitamos? Estad Apl Las Cienc Soc Fac Humanidades Univ Pontif Comillas 2012:24

20. Buchholzer S, Faure F, Tcheremissinoff L, et al (2021) Novel Multidisciplinary Salivary Gland Society (MSGS) Questionnaire: An International Consensus. The Laryngoscope. https://doi.org/10.1002/lary.29731 

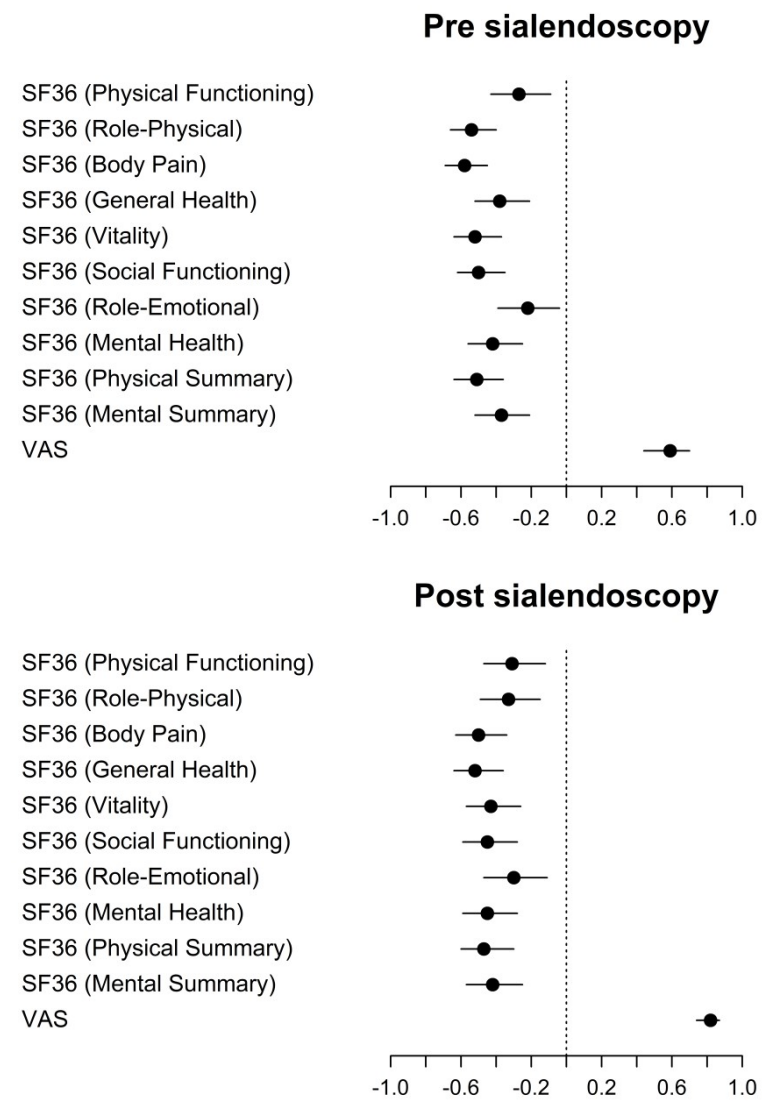

Correlation coefficient

(95\% Cl)

$-0.27(-0.43,-0.09)$

$-0.54(-0.66,-0.40)$

$-0.58(-0.69,-0.45)$

$-0.38(-0.52,-0.21)$

$-0.52(-0.64,-0.37)$

$-0.50(-0.62,-0.35)$

$-0.22(-0.39,-0.04)$

$-0.42(-0.56,-0.25)$

$-0.51(-0.64,-0.36)$

$-0.37(-0.52,-0.21)$

$0.59(0.44,0.70)$

Correlation coefficient $(95 \% \mathrm{Cl})$

SF36 (Physical Functioning) SF36 (Role-Physical) SF36 (Body Pain) SF36 (General Health) SF36 (Vitality) SF36 (Social Functioning) SF36 (Role-Emotional) SF36 (Mental Health) SF36 (Physical Summary) SF36 (Mental Summary) VAS

$$
\begin{aligned}
& -0.31(-0.47,-0.12) \\
& -0.33(-0.49,-0.15) \\
& -0.50(-0.63,-0.34) \\
& -0.52(-0.64,-0.36) \\
& -0.43(-0.57,-0.26) \\
& -0.45(-0.59,-0.28) \\
& -0.30(-0.47,-0.11) \\
& -0.45(-0.59,-0.28) \\
& -0.47(-0.60,-0.30) \\
& -0.42(-0.57,-0.25) \\
& 0.82(0.74,0.87)
\end{aligned}
$$

\section{Hosted file}

Table 1.docx available at https://authorea.com/users/323343/articles/553944-validation-of-aspanish-chronic-obstructive-sialadenitis-quality-of-life-questionnaire

\section{Hosted file}

Table 2.docx available at https://authorea.com/users/323343/articles/553944-validation-of-aspanish-chronic-obstructive-sialadenitis-quality-of-life-questionnaire

\section{Hosted file}

Table 3.docx available at https://authorea.com/users/323343/articles/553944-validation-of-aspanish-chronic-obstructive-sialadenitis-quality-of-life-questionnaire 